\title{
The effect of configural orientation on perceived trajectory in apparent motion
}

\author{
DENNIS R. PROFFIT and DAVID L. GILDEN \\ University of Virginia, Charlottesville, Virginia \\ MARY K. KAISER \\ NASA Ames Research Center, Moffett Field, California \\ and \\ SUSAN M. WHELAN \\ University of Virginia, Charlottesville, Virginia
}

\begin{abstract}
In three experiments, we investigated apparent motion trajectories for stimuli flashed in different locations and at different orientations. It was found that, when stimuli were presented at different orientations, apparent motion trajectories were curved, although these paths were actually circular for only a restricted range of parameters. Curved apparent motion paths were induced by orientation changes in two stimuli that differed in how their orientation was specified. One was a rectangle (orientation-specific contour); the other was a circle (orientation-specific internal patterning). The following variables influenced the extent of apparent curvature seen: (1) the amount of orientation change presented, (2) the orientation of the stimulus symmetry, (3) the salience of configural orientation, and (4) the gender of the observer.
\end{abstract}

In three experiments, we investigated the question, What determines motion paths in long-range apparent motion when stimuli are presented at different orientations? Existing work on this issue suggests two alternatives, each describable in terms of a minimum principle. Our results suggest that the perceptual system selects paths that are compromises between these alternative minimal solutions. Moreover, we found that configural orientation influences path perception in a manner not predicted by any previously proposed simplifying processes.

The motion paths that are observed in long-range apparent motion depend in part on the orientability of the stimuli. Previously, we discussed the relevance of orientation in real object motions (Proffitt \& Gilden, 1987), and distinguished between two types of dynamical contexts, particle motions and extended body motions. ${ }^{1}$ Apparent particle motions involve object displacements without orientation change. They occur when stimuli consist of such nonorientable shapes as points or circles; however, particle motions also occur when orientable objects undergo displacements without changing their orien-

This research was supported by Air Force Grant AFOSR-87-0238, NASA Grant NCA2-87, and NICHD Grant HD-16195. The second author was supported by NRSA Postdoctoral Fellowship HD-07036 from NICHD. Heiko Hecht, Jeffrey Lande, Ellen McAfee, Mary Riser, David Shulman, and Monica Schnicke helped conduct the experiments. Stephen Jacquot programmed the stimulus displays. Portions of this paper were presented at the Western Psychological Association's 67th annual convention, April 1987. Reprint requests should be addressed to Dennis Proffitt, Department of Psychology, Gilmer Hall, University of Virginia, Charlottesville, VA 22903-2477. tation. These motions are particulate because they are reducible to the motions of objects' centers of mass; object configuration is irrelevant. Apparent extended body motions include orientation changes. They occur whenever an orientable object changes its orientation. Thus, extended body motions involve all of the possible displacements found for particle motions, in conjunction with those motions that yield orientation changes. Object configuration is relevant because an object's center of mass, being a point particle, has no orientation specificity. This categorization of apparent object motions depends not on whether objects are particulate or extended, but rather on the motion context in which they are found. An extended form undergoing a displacement without changing its orientation is participating in a particle motion, whereas the same object undergoing an orientation change is participating in an extended body motion.

In apparent motion situations, this distinction is defined by whether stimuli are presented at different orientations. Apparent extended body motions involve orientation changes, whereas apparent particle motions do not. Minimum principles have been proposed to account for path perception in both types of apparent motion situations.

\section{A MINIMUM PRINCIPLE FOR APPARENT PARTICLE MOTIONS}

Apparent particle motions can be seen when two stimuli appropriately flashed in different locations are (1) pointlike, or (2) extended forms that preserve orientation across displacement. These conditions are illustrated in Panels 
$\mathrm{A}$ and $\mathrm{B}$ of Figure 1. As depicted, the apparent motion paths for these conditions will correspond to the minimal translation for the displacement (Wertheimer, 1912). Without some inherent perceptual constraint, this need not be so. Panel $\mathrm{C}$ of Figure 1 shows a possible motion path for two points flashed in different locations. That such a path is almost never reported suggests that the perceptual system selects a minimal solution in determining apparent particle motions. Unless more complex paths are induced by other means (Shepard \& Zare, 1983), apparent particle motions follow minimal translations for any given displacement.

\section{TWO MINIMUM PRINCIPLES FOR APPARENT EXTENDED BODY MOTIONS}

In apparent extended body motions, the flashed stimuli not only are displaced from each other, but are also flashed with different orientations. Such an event is depicted in Panel A of Figure 2. Two proposals predict the different motion paths that could be seen in such circumstances (Panels B and C). Each of these proposals reflects a different minimum principle.

\section{Information Minimization}

In Panel B of Figure 2, the rectangle is shown moving along an apparent path that corresponds to a single rotation. Chasle's theorem proves that any object displacement and orientation change can be achieved through a unique single rotation. Foster (1975) created an apparent motion display similar to that shown in Figure 2. He re-

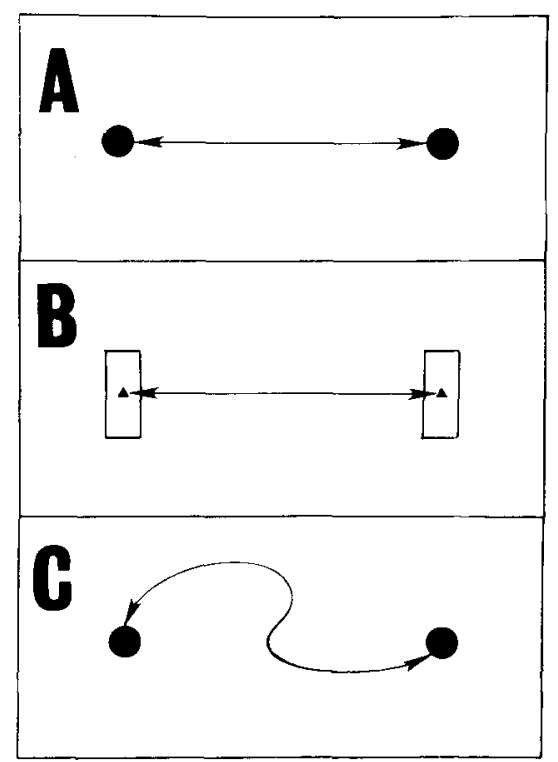

Figure 1. The top and middle panels show the linear trajectories that are typically observed in apparent motion of a point-like stimulus (Panel A) or an extended shape that preserves orientation across displacement (Panel $\mathrm{B}$ ). Panel $\mathrm{C}$ depicts one of the indefinite number of other, more complex, trajectories, which tend not to be reported.

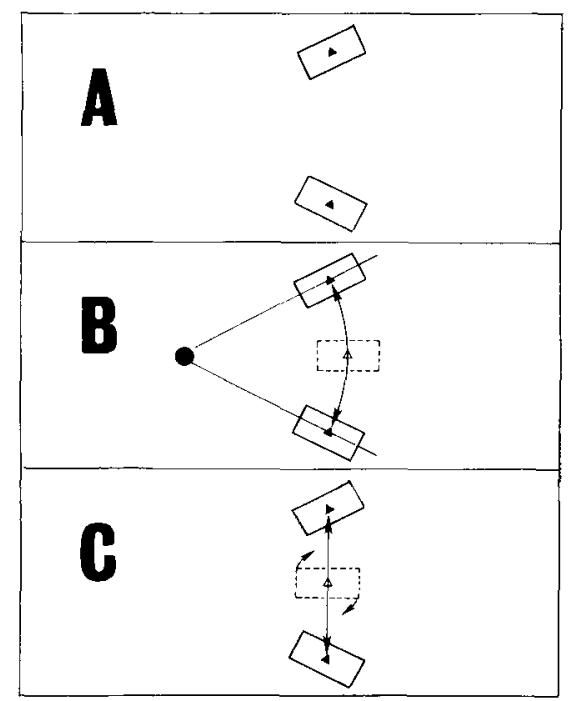

Figure 2. Panel $A$ depicts a situation in which two rectangles are sequentially flashed in different locations and at different orientations. Panel B shows the single circular appanent trajectory predicted by the information minimization principle. Panel $\mathrm{C}$ shows the linear common motion and concomitant rotation apparent trajectories predicted by the motion minimization principle.

quired his observers to place a probe rectangle at various locations and orientations along the apparent motion path of the flashing display. Using this method, he determined the trajectory of apparent motion and found it to correspond to the single-rotation solution.

Shepard (1984) used Foster's (1975) results as support for his proposal that the perceptual system constrains apparent extended body motions by minimizing their descriptions within kinematic geometry. Given 6 degrees of freedom for rigid object motions ( 3 for translation and 3 for rotation), the perceptual system's solution reflects a minimization of the number of motion parameters needed to describe a rigid motion over the presented displacement and orientation change. In essence, Shepard's account suggests that the perceptual system seeks an information minimization on the degrees of freedom in the event.

Restle's (1979) coding theory, although formulated in the context of understanding the perception of real motions, makes identical predictions to those of Shepard (1984). Restle proposed that the motion perception system seeks to minimize the number of information parameters needed to code an event. Given his information coding system, the singular rotation possesses the minimum information code for any concomitant displacement and orientation change.

\section{Motion Minimization}

The above accounts minimize the information content of apparent extended body motions; however, they do not derive minimal solutions relative to the amount of apparent motion perceived. Panel $\mathrm{C}$ of Figure 2 shows the 
minimal motion solution for the event depicted in Panel A. In general, the minimal motion for an object, given a displacement and orientation change, is a rotation about its centroid and a translation of the centroid. Any other motion, such as that predicted by the information minimization accounts, causes the object to move a greater distance (as defined by the integration of the trajectories for all object points).

Cutting and Proffitt (1982) proposed a motion minimization account for real motions in which the perceptual system attempts to achieve a minimization of both relative motions and common motion. Relative motions (rotations) are minimized when they occur around the object's centroid. Common motion is minimized when it corresponds to a linear path taken by some point within the configuration. Both of these minimizations are achieved with centroid-relative rotations and concomitant translation for apparent extended body motions.

Farrell (1983) assessed the minimum stimulus onset asynchrony (SOA) for apparent extended body motion displays having various displacements and orientation changes. She then compared her results with two models: Singular rotation and linear displacement with concomitant rotation. (Constant velocity was assumed for both rotations and translations.) The best fit for her results was the latter model, that is, the minimal motion solution.

\section{OVERVIEW OF THE STUDIES}

We have discussed two accounts for perceived path perception in apparent extended body motions. Some empirical support for both approaches does exist. Foster's (1975) study supports the information minimization account; however, he employed stimuli with a very restricted range of parameters. Farrell's (1983) study supports the motion minimization approach; however, her study did not include a direct assessment of path perception, but rather, perceived motion paths were inferred from minimum SOA assessments under the assumption of constant velocities.

In the present experiments we used a direct assessment of apparent path perception over a wide range of stimulus parameters. Experiment 1 was a replication of Foster's (1975) study, using a somewhat different methodology. The results of this study showed that our technique yielded results similar to Foster's. In Experiment 2 we examined a very wide range of stimulus parameters. In particular, we examined not only the magnitude of orientation change, but also the orientation of configural symmetry to the direction of orientation change. Both of these variables proved to be influential in determining apparent path perception. Finally, in Experiment 3, we examined the influence of configural orientation change produced by internal patterning, as opposed to external boundary contour. Internal contours were found to produce apparent extended body motion effects, but of a lesser magnitude, and only for male observers. In summary, our results did not support either minimum principle, but rather suggested a more complex influence of configural orientation on apparent extended body motions.

\section{EXPERIMENT 1: REPLICATION OF FOSTER'S (1975) RESULTS}

The experimental apparatus and techniques that we used in presenting apparent motion displays differed in several respects from those employed by Foster (1975). In this initial experiment, we sought to show that these differences would not prevent a replication of Foster's basic finding; that is, that for the stimuli he presented, our subjects would perceive circular apparent motion paths.

\section{Method}

Subjects. Twelve observers, 6 males and 6 females, participated in this experiment. Acquaintances of the authors and faculty and graduate students from various University of Virginia departments served as observers. Observers were tested individually. One additional observer was deleted from the study due to an inability to comprehend the task.

Stimuli. All stimuli were viewed on a Tektronix 4129 3-D Color Graphics Workstation with a high-resolution (4 lines $/ \mathrm{mm}$ ) display screen $30 \mathrm{~cm}$ high $\times 35 \mathrm{~cm}$ wide. The stimuli consisted of two fixed-position blinking test rectangles and a probe rectangle that could be moved and turned off and on by the observer. As shown in Figure 3, the spatial organization of the test rectangles is defined by three parameters: the angle of a line, equidistant at every point to the centers of the test rectangles (we call this line the system axis), relative to the horizontal screen axis (beta); the angle that a ray extending from the origin of the single rotation solution to a test rectangle center (we call this ray the rectangle arm) makes with respect to the system axis (theta); and the angle that the rectangle's major axis makes with respect to the rectangle arm (alpha). For any given stimulus, theta and alpha are the same for both test rectangles. The orientation of each test rectangle was further specified by coloring the lines $\mathrm{AB}$ and $\mathrm{CD}$ red and blue, respectively. Rectangle dimensions $(4.1 \times 0.8 \mathrm{~mm})$ and the distance between test rectangle centers $(13.3 \mathrm{~cm})$ were determined in pilot testing to optimize stable path perception.

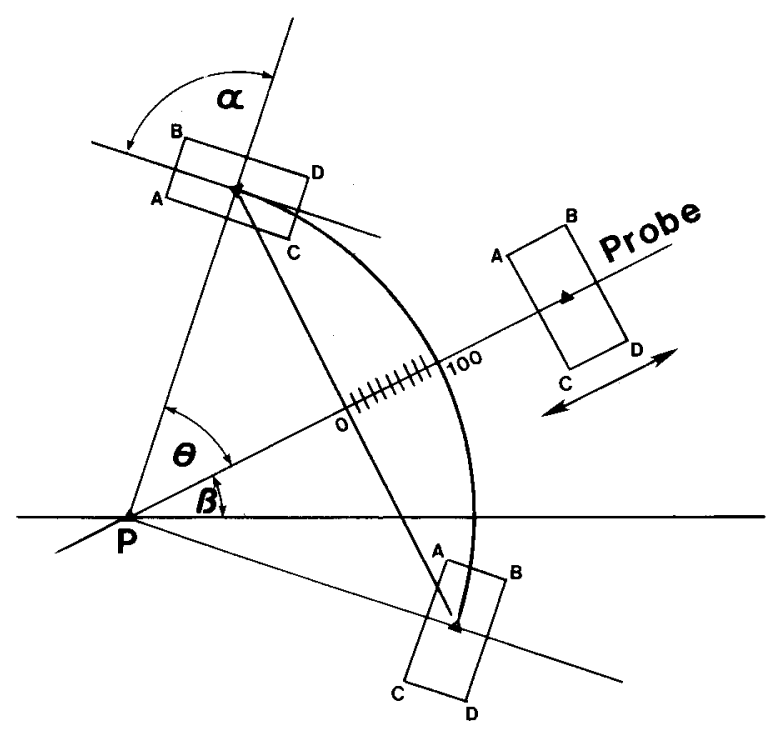

Figure 3. Stimulus parameters. 
The test rectangles were positioned such that the point of intersection $(\mathrm{P})$ between the system axis and the rectangle arm was near the center of the screen. In order to lessen the likelihood that the screen center would serve as a cue for motion perception, the placement of $P$ for each stimulus varied randomly within a 4-cm circle at the screen's center. At the viewing distance of approximately $60 \mathrm{~cm}$, the absolute separation of the test rectangle centers subtended an angle of about $12.6^{\circ}$.

In addition to the test rectangles, the stimuli included a probe rectangle that could move continuously along the system axis. The orientation and coloring of the probe rectangle on the system axis was identical to the orientation and coloring of the test rectangles on their respective arms. The probe rectangle could be turned off and on as the observer desired. At the commencement of each stimulus presentation, the probe rectangle was either on the left or on the right border of the screen, but turned off. In order to see the probe rectangle, the observer had to turn it on.

The blinking sequence of the test and probe rectangles was defined by the duration of each rectangle and the interstimulus interval (ISI). The blinking sequence was as follows: test 1 , probe, test 2 , probe, repeat, where the stimulus durations were $70 \mathrm{msec}$ and the ISI in each case was $180 \mathrm{msec}$. The ISI between presentations of the two fixed test rectangles was the same whether the probe rectangle was on or off. The ISIs and the stimulus durations were determined from extensive piloting.

Comparison with Foster's (1975) stimulus presentation. The primary difference between our stimulus presentation and that of Foster (1975) was in the implementation of the probe rectangle. The probe rectangle in Foster's display was not constrained to move along the system axis, but could move to any part of the screen. In Foster's design, the experimenter would constrain the horizontal position of the probe rectangle and the observer would adjust both the probe's vertical position and orientation about its center until it lay on the perceived path. The apparent motion paths in Foster's study were resolved with three or four points, each point specifying not only spatial position, but also the local tangent vector derived from the probe's orientation. In our design, the probe rectangle was constrained to move along the system axis with a fixed orientation. Our paths were therefore resolved by a single spatial point that contained the local tangent vector as a boundary condition. A second difference is that our probe blinked in synchrony with the test rectangles, whereas the probe in Foster's experiment was on continuously. This feature allowed our probe rectangle to participate in the apparent motion. To minimize the influence of the probe's position on observers' responses, we required that the observers turn the probe rectangle off and on to ensure that the path with the probe on was identical to the path with the probe off.

Design. Foster (1975) presented eight stimuli. These stimuli were very similar; we chose the four most diverse for use in this experiment and used the rest in practice trials. The angle parameters (alpha/theta) used in the experiment were $0^{\circ} / 20^{\circ}, 0^{\circ} / 22^{\circ}, 0^{\circ} / 24^{\circ}$, and $90^{\circ} / 20^{\circ}$, for Test Stimuli 1 through 4 , respectively. The four practice stimuli were viewed twice, in two blocks. In the first block, the presentation order of the four practice stimuli was random, as was the initial placement of the probe rectangle. In the second block, the four practice stimuli were again presented in random order, but the initial placement of the probe rectangle was opposite what it had been in the first block. The presentation of the four test stimuli was accomplished in the same way, except that two additional blocks were added. In these final two blocks, the initial probe placement was always opposite what it had been in the previous display for each stimulus. In other words, if in the first block a given stimulus had the probe initially on the left, in the subsequent three blocks the probe would be placed initially on the right, left, and right, respectively.

Procedure. The experimenter briefly described apparent motion. The observers were instructed to attain good path perception before turning the probe rectangle on. Once a stable path was perceived, they were to turn on the probe and move it onto the path. They were then to turn the probe off, checking that the path with the probe on was the same as the path with the probe off. The observers were given a rest period halfway through the experiment. During this period they completed a task that had no relation to path perception.

\section{Results and Discussion}

The observers' probe placements were scored on a 0 to 100 equal-interval scale, with 0 representing a straight trajectory and 100 representing a circular trajectory. The average probe placements for the four test stimuli are shown in Figure 4. Our results replicated those of Foster

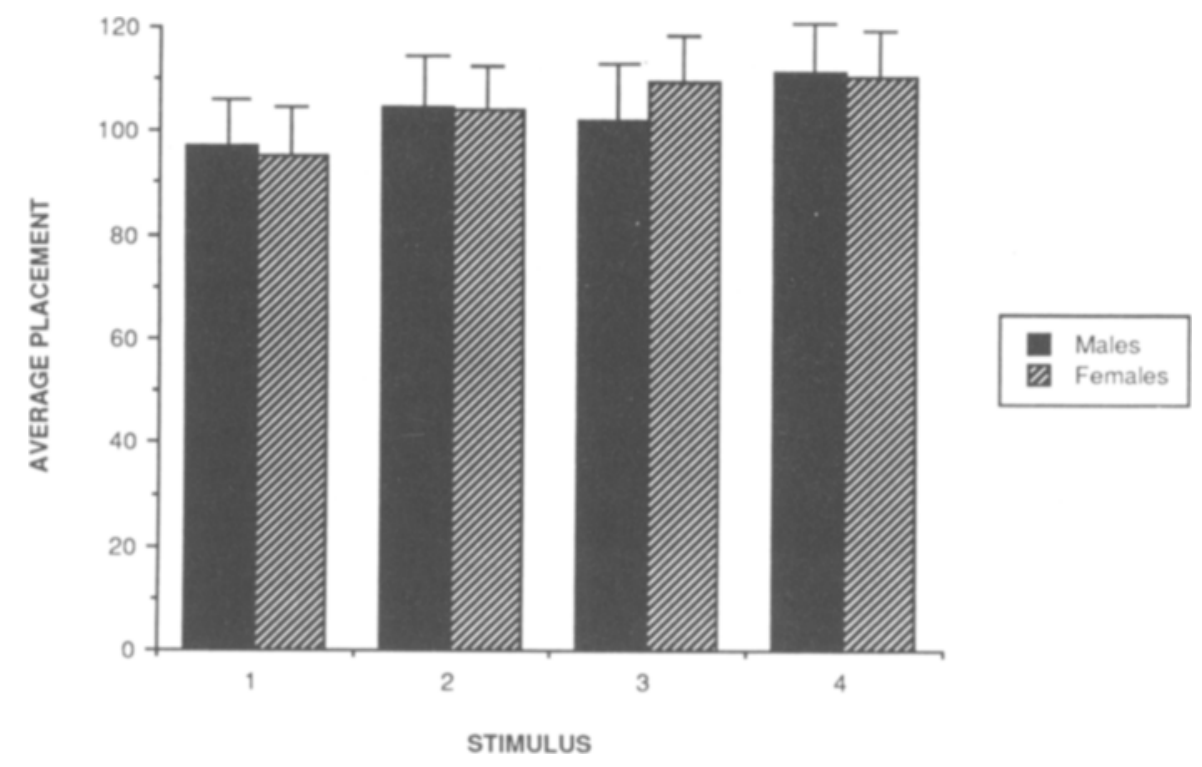

Figure 4. Results for Experiment 1. A placement of 0 corresponds to linear trajectory and a placement of 100 corresponds to a circular trajectory. Vertical bars indicate $+1 S E M$. 
(1975); the observers' probe placements indicated that they saw circular trajectories. Only for the fourth stimulus did the mean probe placement differ significantly from the circular trajectory position, and then it was in the direction away from the minimal motion alternative $[t(11)$ $=2.50, p<.05]$. No effect was noted for gender or the initial position of the probe.

\section{EXPERIMENT 2: THE DEPENDENCE OF PATH PERCEPTION UPON CONFIGURAL ORIENTATION}

The stimuli used by Foster (1975) were of a very specific nature: The principal axis of the test rectangles was aligned with (our Stimuli 1-3) or perpendicular to (Stimulus 4) the rectangle arm. In extensive piloting of path perception, we found that circular paths were, in fact, perceived only when these alignments were realized, and that the former alignment-the principal axis coinciding with the rectangle arm-yielded the most consistent curved path perception. For other alpha orientations, observers reported that the rectangle's centroid appeared to move more in a straight line (with a concomitant rotation about the centroid), and that this path was relatively unstable; that is, there was no place where they felt the probe rectangle definitely belonged. It appeared that correlations existed between circular path perception, ease and robustness of the perception, and the orientation of the principal axes on the rectangle arm.

An additional influence was found for the theta parameter. For large values of theta, say theta $>60^{\circ}$, the perception of circular paths was increasingly difficult. This phenomenon also has a straightforward geometric interpretation. The spatial separation on the screen between the straight line path and the rigid rotation circular path is a monotonically increasing function of theta. In other words, the length of the straight line path is constant for all stimuli, whereas the length of the rigid rotation circular path increases with theta. Observers were disinclined to perceive circular paths that took long excursions from the straight line path. Furthermore, the most easily perceived circular paths were those with modest values of theta, say theta $\sim 30^{\circ}$.

In this experiment, we attempted to provide quantitative measures for these informal observations. In addition to standardized placement scores, these measurements included the raw score denoting the placement of the probe rectangle, a goodness rating for ease and stability of path perception, and the time taken by the observer to place the probe rectangle on the apparent motion path.

\section{Method}

Subjects. Twelve observers, 6 males and 6 females, participated in the experiment. They were recruited from introductory psychology courses and received course credit for their participation. None of the observers was aware of the study's purpose, and none had participated in the first experiment.

Stimuli. The construction and presentation of the stimuli were as described for Experiment 1, the only difference here being in the values of alpha, beta, and theta that were employed. Beta (the angle that the system axis makes with the horizontal screen axis) was chosen randomly from a set of four values $\left(45^{\circ}, 135^{\circ}, 224^{\circ}\right.$, and $315^{\circ}$ ) with the constraint that beta differ on successive trials. The alpha-theta plane was sampled with a $7 \times 6$ factorial design: alpha $=0^{\circ},-15^{\circ}, 30^{\circ},-45^{\circ}, 60^{\circ},-75^{\circ}, 90^{\circ}$; theta $=15^{\circ}, 30^{\circ}$, $45^{\circ}, 60^{\circ}, 75^{\circ}, 90^{\circ}$. (Alphas varied in alternating signed increments of $15^{\circ}$ from $0^{\circ}$, inasmuch as relative alignment with respect to $0^{\circ}$ was the variable of interest.)

Each trial was timed, so that the difficulty that observers had with probe placement could be measured. Timing began with the first keystroke that moved the probe rectangle from its initial position on a screen border and ended when the observer pressed a key indicating that there were no further adjustments he/she wished to make on the probe's position for that stimulus. The observers were not told that the procedure was being timed.

Following the observer's completion of the probe placement task, a goodness scale appeared on the bottom of the screen. The observer was asked to rate the goodness of his/her path perception, on the basis of his/her confidence in the placement of the probe rectangle and the clarity of path perception. This was accomplished with a thumbscrew that moved a marker along the goodness scale. The goodness rating was completed with the entire stimulus display presented on the screen.

Design. A mixed-model factorial design was employed, with gender as a between-subjects factor, and alpha, theta, and initial probe position as within-subjects factors. As mentioned above, there were seven levels of alpha and six levels of theta. Initial probe position had two levels (left and right).

Procedure. The procedure was identical to that of Experiment 1.

\section{Results and Discussion}

The observers' standardized probe placement scores were examined for outliers. Scores of less than -100 or greater than 200 were reset to these boundaries. (Additional analyses that excluded outliers yielded findings virtually identical to those reported below.)

As predicted, both the alpha and theta parameters affected the observers' probe placement. Figure 5 shows average placement as a function of alpha. As the principal axis of the rectangle became less aligned with the rectangle arms, less circular trajectories were perceived $[F(6,60)=7.91, p<.001 ;$ a linear trend accounts for $95.2 \%$ of the variance]. Similarly, as theta increased, probe placement again indicates that observers saw less circular trajectories. The average placement as a function of theta is shown in Figure 6. Again, this effect was highly significant $[F(5,50)=14.96, p<.001]$ and primarily linear $(75.7 \%$ of the variance accounted for by linear trend analysis). There was no significant interaction between the alpha and theta parameters.

As can be seen in Figure 5, apparent trajectories became progressively more shallow as alpha increased; moreover, an examination of responses to individual stimuli showed that circular paths were not observed for any of the stimuli with alphas of $90^{\circ}$. This result indicates a failure to replicate the findings for Stimulus 4 in Experiment 1. In Experiment 1, this stimulus with an alpha of $90^{\circ}$ was seen to move along a circular path. We suspect that the circular path perceived for Stimulus 4 in Experiment 1 was primed by the robust circular paths observed for the other three stimuli, which had alphas of 


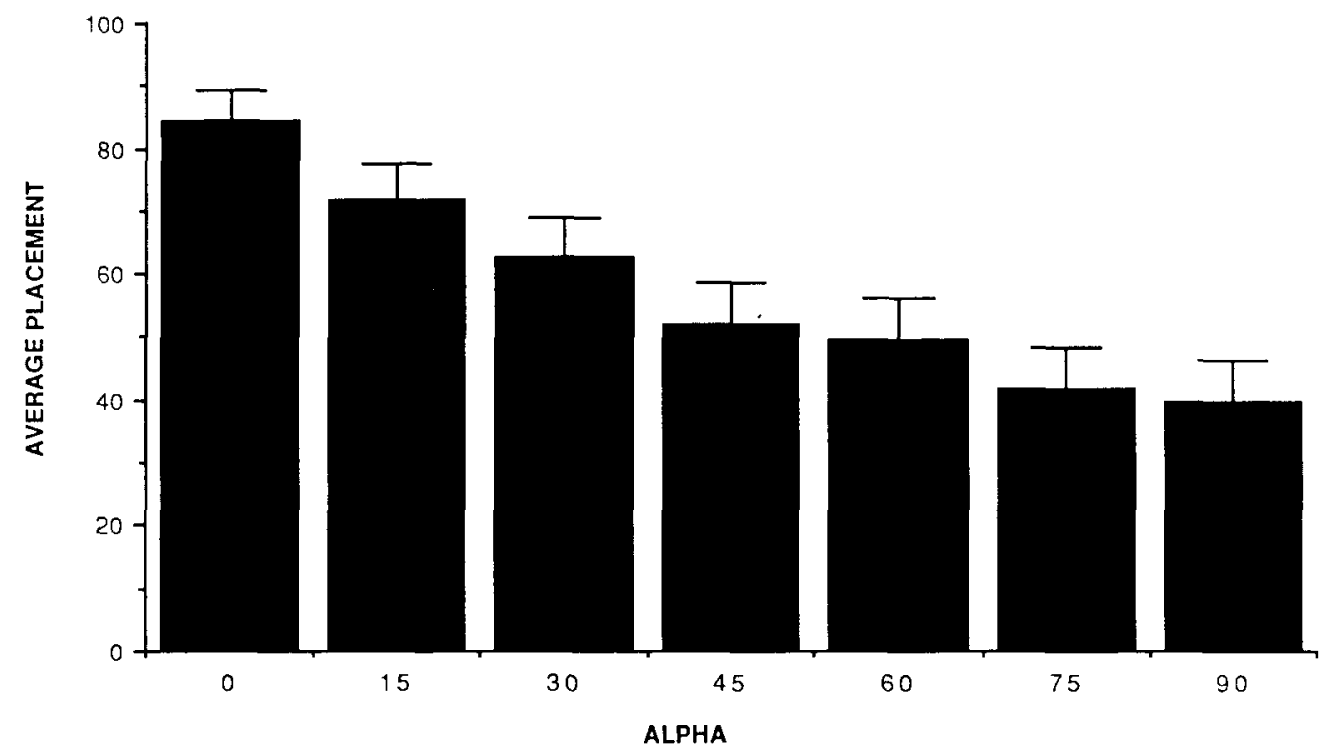

Figure 5. Effect of object orientation (alpha) in Experiment 2. A placement of 0 corresponds to linear trajectory and a placement of 100 corresponds to a circular trajectory. Vertical bars indicate $+1 S E M$.

$0^{\circ}$. (The priming of circular paths is discussed under Experiment 3 , in which such a "set effect" was clearly demonstrated.)

Probe placement differed as a function of gender $[F(1,10)=15.98, p<.005]$, with males preferring more curved trajectories (mean placement was 68.5 for males, 45.9 for females). (This gender effect was not due to extreme scores by only 1 or 2 observers; only 1 male observer's average placement fell within the range of mean placements for the female observers.) Placement did not differ significantly for the two initial probe positions.
The above analyses employed the standardized placement scores; that is, the distance between the straight and circular paths was scaled to a 100 -unit metric. If the raw placement scores (i.e., the distance from a straight trajectory in pixel units) are used, a somewhat different picture emerges. The alpha effect is quite similar, except for the scaling factor. As with the standardized placement scores, trajectories become less curved as the alpha parameter increase $[F(6,60)=14.51, p<.001$; linear trend accounts for $94.9 \%$ of the variance]. The effect for theta, however, is reversed. More absolute deviation from

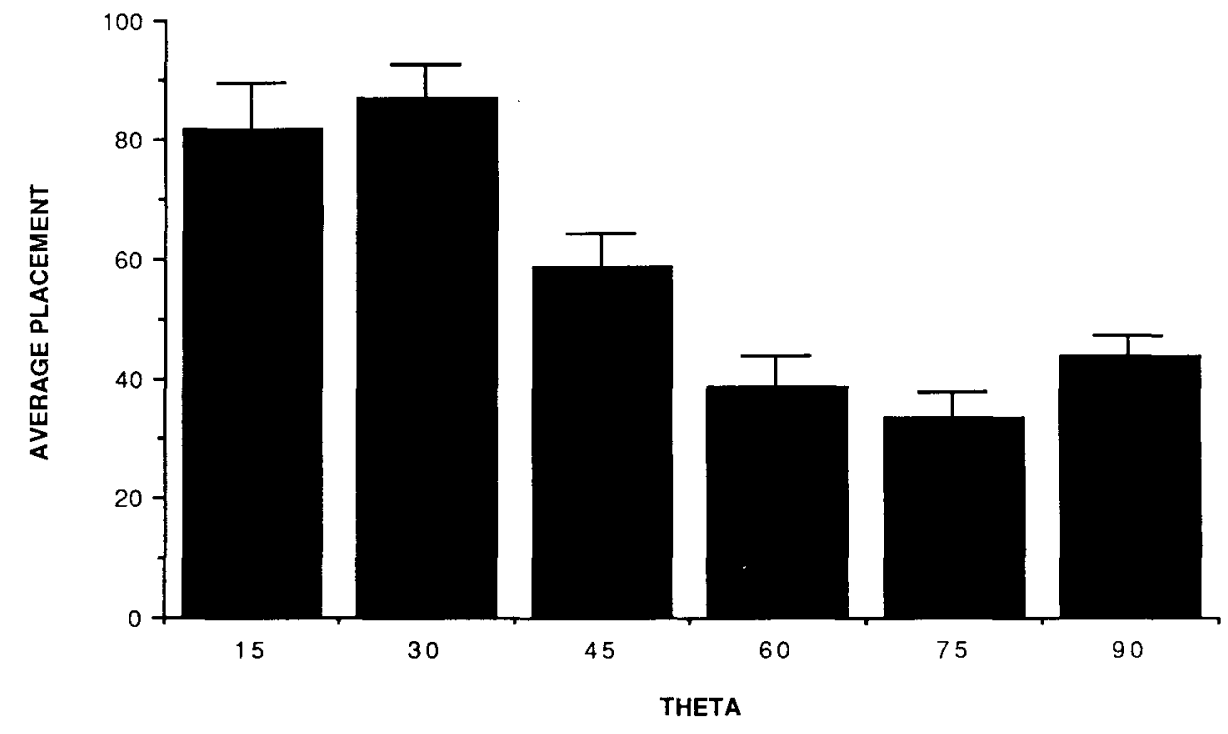

Figure 6. Effect of rotational magnitude (theta) in Experiment 2. A placement of 0 corresponds to linear trajectory and a placement of 100 corresponds to a circular trajectory. Vertical bars indicate +1 SEM. 


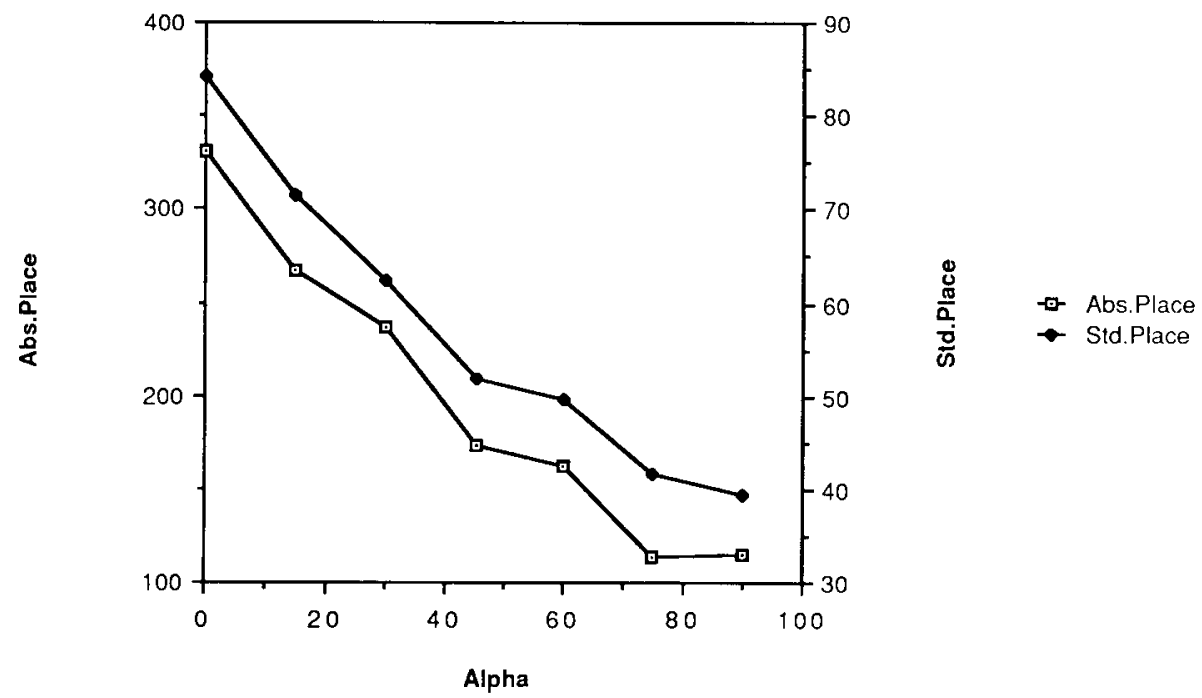

Figure 7. Results for Experiment 2 plotted as standardized (Std.) versus raw (Abs.) placement scores for alpha.

a straight trajectory is seen for larger values of theta because, as indicated, the number of pixel units between the straight and circular paths increases as a function of theta. The standardized versus raw score functions for alpha and theta are shown in Figures 7 and 8, respectively.

The effect of theta on path perception is best understood by interpreting the results in terms of both standardized and raw placement scores. Standard scores specify where trajectories fall between the theoretical boundaries of straight and circular paths. Raw scores define the trajectories' absolute amplitudes of curvature relative to the straight line minimum. Examination of both scores reveals that, as theta increased, perceived motion paths became less circular, although the absolute deviation of these trajectories from a straight line path increased.
Only circular trajectories achieve information minimization. As is the case for straight paths, all other curvatures require a second motion component to achieve the displayed orientation change. On the other hand, only straight trajectories achieve motion minimization. Thus, the influence of theta compromises both minimum principles.

Observers' goodness ratings for their probe placements were normalized within observers. These normalized goodness ratings had a significant negative correlation with response time; that is, the observers were less satisfied with the placements that took longer $(r=-.32$, $p<.01$ ). Goodness ratings also had a significant correlation with standardized probe placement; trajectories with more curvature received higher ratings $(r=.171$,

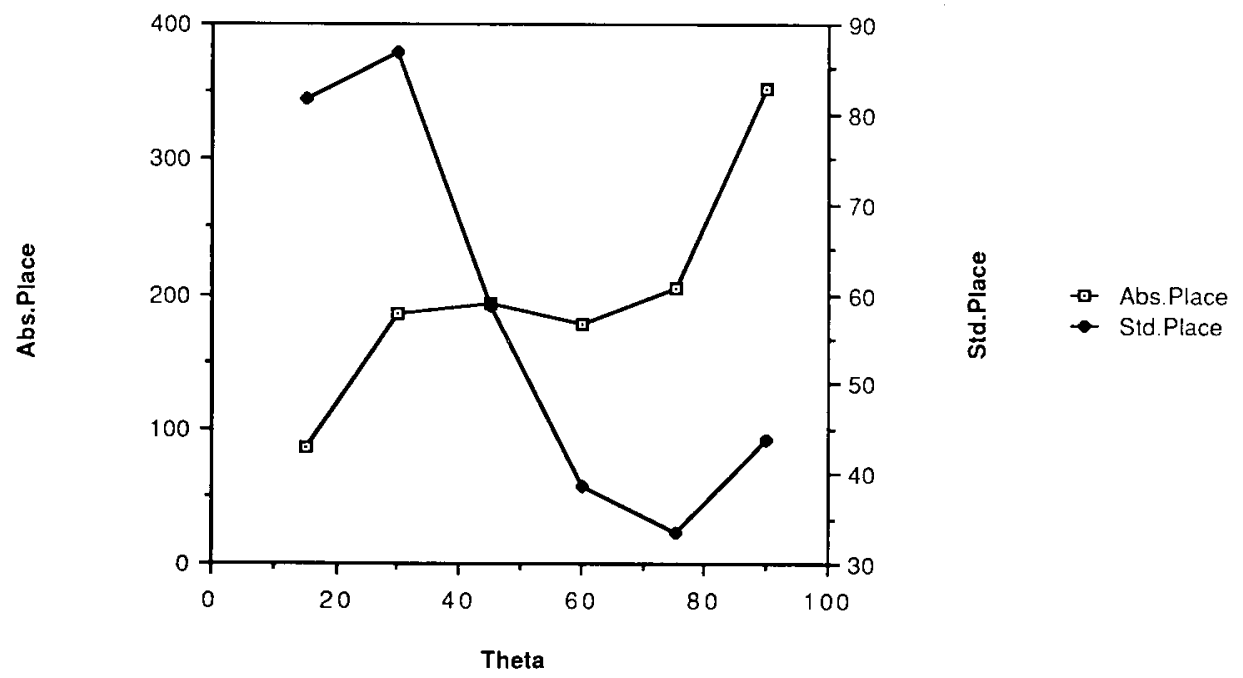

Figure 8. Results for Experiment 2 plotted as standardized (Std.) versus raw (Abs.) placement scores for theta. 
$p<.05$ ). Probe placement and response time were not related.

Goodness ratings also correlated with stimulus parameters alpha and theta. Larger values of alpha received lower goodness ratings $(r=-.17, p<.05)$, as did higher values of theta $(r=-.18, p<.05)$. Thus, as expected, those stimuli that produced less curved trajectories were judged as less satisfactory.

The results for the goodness ratings indicate that apparent circular motion paths were more robust, in the conditions that favored their perception, than were the more shallow trajectories that other stimulus conditions induced. This finding suggests that information minimization may be preferred by the perceptual system, although its induction requires a restricted range of alpha and theta stimulus parameters.

\section{EXPERIMENT 3: DO ORIENTATIONAL CHANGES IN INTERNAL PATTERNING SUFFICE TO INDUCE CURVED PATH PERCEPTION?}

In Experiment 2 we found that curved path perception was evident throughout most of the alpha-theta plane, and that for values of alpha near $0^{\circ}$ (major axis of the rectangle aligned with the rectangle arm), the curved paths were nearly circular (for values of theta $\sim 30^{\circ}$ ). Rectangle orientation is apparently sufficient to induce the perception of curved paths. Orientation information in Experiments 1 and 2 was manifest through the boundary contour. Orientation information may also be imparted through internal patterning. An appropriate comparison for the unpatterned rectangle is a disk that provides orientation information only through an internal pattern. In Experiment 3 we examined whether patterned disks would induce the perception of curved paths.

\section{Method}

Subjects. Twenty-four University of Virginia students participated in this experiment for credit in an introductory course in psychology. All observers were naive as to the purpose of the study and had not participated in previous experiments.

Stimuli. The stimulus presentation was identical to that of Experiments 1 and 2 except that two disks were presented instead of rectangles. The disks were $2 \mathrm{~cm}$ in diameter. The patterned disk had six equally spaced parallel lines threading its interior. To further specify the orientations of the patterned disk, the lines in one semicircle were colored blue and the lines in the other semicircle were colored red. The outline of the disk was magenta. The region between the lines was the uniform gray of the background. The unpatterned disk was separated from the background only by the magenta outline. The patterned disk was viewed at two values of alpha $\left(0^{\circ}\right.$ and $\left.90^{\circ}\right)$ at the unique value of theta $=30^{\circ}$. These stimulus parameters were quite similar to those employed by Foster (1975). In all cases the probe disk was identical to the test disks.

Design. The design was similar to that of Experiment 1. A block using the patterned disk consisted of presentations of the disk in both alpha conditions and with the initial probe placement on both the left and the right. A block using the unpatterned disk varied only the left/right initial probe placement. In all cases, two blocks were viewed by each observer. For those observers viewing both the patterned and unpatterned disks, the patterned disk block was viewed first.

Procedure. The observers were divided into two groups, with 6 males and 6 females in each group. One group viewed both patterned and unpatterned disks (no-pattern-exposed); the other viewed only unpatterned disks (no-pattern-naive). As in Experiments 1 and 2 , the observers were briefed on apparent motion. Those who saw patterned disks were given practice with them and then were given the blocks with the patterned disks. Following completion of all viewings of the patterned disk, the observers were given a rest period during which they completed a distractor task. Finally, these observers viewed the blocks of unpatterned disks. Observers who saw only unpatterned disks were given a block of practice and then the two test blocks. Response times and ratings of paths were recorded as in Experiment 2.

\section{Results and Discussion}

Average probe placement for the three viewing conditions (pattern, no-pattern-exposed, and no-pattern-naive) are shown for males and females in Figure 9. The withinsubjects comparison for stimulus pattern (i.e., pattern vs. no-pattern-exposed) was highly significant for the males $[t(5)=10.44, p<.001]$, but not for the females $[t(5)$ $=0.02]$. Likewise, the between-subjects test for set effects (i.e., no-pattern-exposed vs. no-pattern-naive) was significant for males $[t(10)=11.18, p<.001]$ but not for females $[t(10)=0.03]$. Thus, the males perceived curved trajectories for the patterned disks, and demonstrated a tendency to see unpatterned disks as curving when they were viewed after the patterned disks. For these subjects, viewing the patterned disks primed the perception of curved paths in subsequent viewing of unpatterned disks. Females, like the no-pattern-naive males, saw the disks travel along straight trajectories.

For the patterned disks, goodness ratings were related to response time $(r=-.21, p<.05)$ and placement score $(r=.22, p<.05)$. Thus, as with the rectangular stimuli, observers were more satisfied with responses that resulted in more curved trajectories and took less time to place. Goodness ratings did not correlate significantly with placement for the unpatterned disks, and correlated with response time only for the observers who had viewed the patterned disks $(r=-.37, p<.01)$.

\section{CONCLUSION}

Apparent extended body motions follow paths that are not predicted by information minimization or motion minimization principles. In general, curved trajectories are seen when orientable stimuli are flashed in different locations and at different orientations, but these common motion paths are circular only for a restricted range of orientation parameters. The following variables influence the extent of curvature seen: (1) the amount of orientation change presented (theta), (2) the orientation of the stimulus relative to an axis of symmetry (alpha), (3) the salience of configural orientation, and (4) the gender of the observer.

Only small orientation changes yield circular apparent motions. The greater the orientation difference be- 


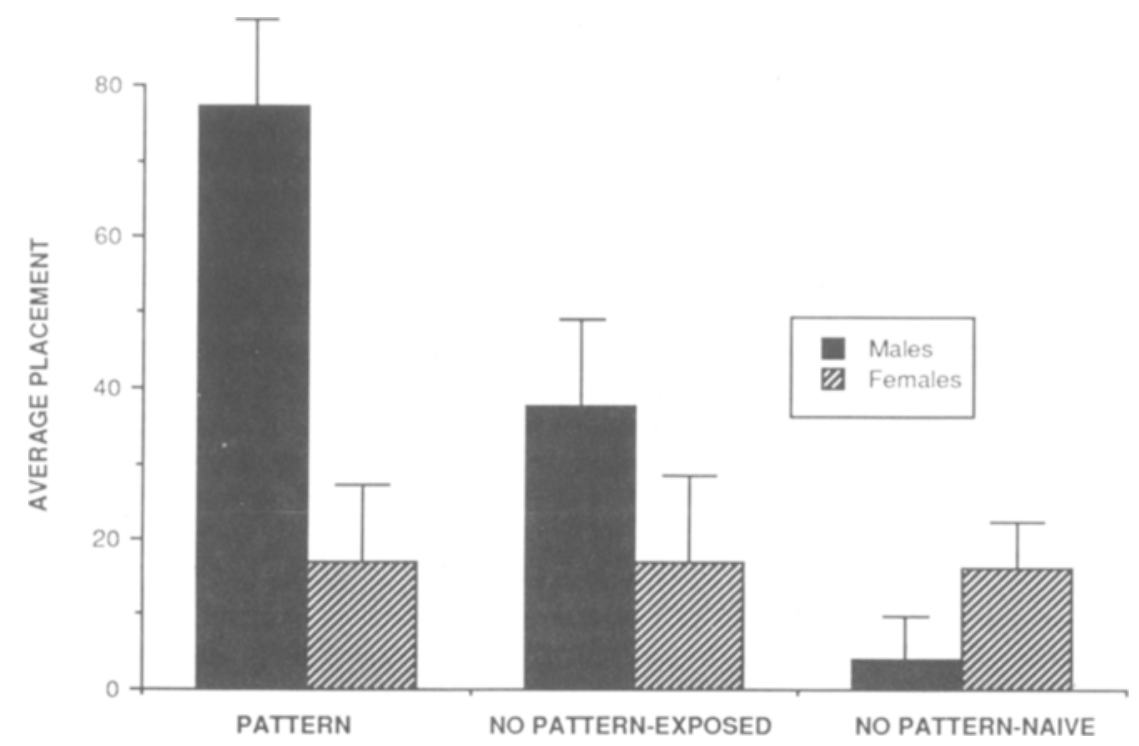

Figure 9. The results of Experiment 3, showing the perceived trajectories of flashed disks having internal orientation specification. A placement of 0 corresponds to linear trajectory and a placement of 100 corresponds to a circular trajectory. Vertical bars indicate $+1 S E M$.

tween two configurations flashed in different locations, the less circular is the apparent motion seen between the two locations. Large orientation changes produce trajectories that are closer to being straight lines than circular arcs, although the absolute distance of these shallow curves from straight line paths is actually greater.

Stimulus conditions that yield highly circular paths are judged as looking better (as assessed by goodness rating), and determination of these trajectories takes less time than determination of trajectories seen with more linear common motion paths. It seems that orientation change induces the perception of curved paths, and that the goodness of these paths is related to the degree to which they are circular.

As Farrell's (1983) study showed, the absolute extent of apparent curvature is related to SOA. Had we used longer or shorter SOAs, the degree of apparent curvature would surely have increased or decreased for particular magnitudes of orientation change (theta). However, our pilot work strongly suggests that the influence of SOA is limited to small and intermediate values of theta. Using the most favorable alpha parameters, none of the authors have been able to achieve the perception of a circular path for theta values greater than $60^{\circ}$. Moreover, the influence of alpha on perceived curvature demonstrates that extent of apparent curvature is not simply a function of time.

The orientation of stimulus symmetry affects apparent extended body motions. When the rectangle's major symmetry axis was aligned with the rectangle arm (the ray passing through the rectangle's centroid and the origin for single circular rotation), more circular paths were seen than at other orientations. The greater the misalignment (alpha), the shallower were the perceived paths.
The major axis of a rectangle defines its orientation, and thus serves as a functional part of the configuration (Rock, 1973). Orientation change in a rectangle is seen by noting changes in its principal axis. When this axis is aligned with the rectangle arm, it effectively "points" at the origin of the single rotation motion path. This pointing, we believe, serves to prime the perception of more circular paths.

Obviously, the particular pattern of results that we obtained for alpha are specific to the rectangular stimulus that we employed. It is likely that changing the rectangle's aspect ratio would yield somewhat different effects. Surely, changing the aspect ratio to that of a square would cause more circular paths to be seen at orientations of $0^{\circ}$ and $90^{\circ}$.

The salience of configural orientation affects apparent extended body motions. We interpret the reduced curvature found for the patterned disks, as compared with that found for the rectangles, as indicating that the degree of apparent curvature is proportional to the salience of stimulus orientation. Further support for this interpretation is needed, of course, inasmuch as only two stimuli were compared.

It is important to note that curved path perception can be induced solely through internal patterning. Consistent with recent findings (Green, 1986; Watson, 1986), these results show that apparent motion can occur between stimuli that share predominantly high-spatial-frequency information. Unique to our study is the finding that orientation influences on path perception can be produced without orientation change's being expressed in boundary information.

Male observers tend to see much more curvature in apparent extended body motions than do females. This was 
found to be the case in both Experiments 2 and 3. We suspect that Experiment 1 yielded too little variability for gender to have an influence. In Experiment 3, female observers were not influenced by the internal patterning and reported almost straight paths in all conditions.

At present, we choose not to speculate on the significance of these gender effects. They are certainly highly robust, and may indicate intrinsic gender differences in the processing of spatial information. Particularly intriguing in this regard is the complete absence, among females, of an influence of internal patterning on path perception. Whatever the cause of these gender effects, their presence is a reason to avoid using only one gender in apparent motion research.

In summary, the present experiments show that the paths perceived in apparent extended body motions tend to follow curved trajectories. The extent of curvature depends on the amount of orientation change present, configural properties of the stimulus, and the gender of the observer. The perceptual constraints that determine path perception in such situations are influenced by all of these variables. Neither of the proposed minimum principles predicts this pattern of results. That trajectories tend always to curve in extended body apparent motions is incompatible with the predictions of a motion minimization principle. That the degree of curvature approximates a circle for only a restricted range of stimulus parameters is inconsistent with the predictions of an information minimization principle. The perceptual system selects trajectories that are compromises between these minimal solutions; for most stimulus conditions, apparent motion paths are neither straight nor circular. An information minimization principle predicts the perceived goodness of apparent extended body motions, but it reflects much more weakly on the form of the inherent perceptual constraints that determine their perceived trajectories.

\section{REFERENCES}

Cutting, J. E., \& Proffitt, D. R. (1982). The minimum principle and the perception of absolute, common, and relative motions. Cognitive Psychology, 14, 211-246.

FARRELL, J. E. (1983). Visual transformations underlying apparent movement. Perception \& Psychophysics, 33, 85-92.

Foster, D. H. (1975). Visual apparent motion and some preferred paths in the rotation group $\mathrm{SO}(3)$. Biological Cybernetics, 18, 81-89.
GREEN, M. (1986). What determines correspondence strength in apparent motion? Vision Research, 26, 599-607.

Proffitt, D. R., \& Gilden, D. L. (1987). Understanding natural dynamics. Manuscript submitted for publication.

Restle, F. (1979). Coding theory and the perception of motion configurations. Psychological Review, 86, 1-24.

Rock, I. (1973). Orientation and form. New York: Academic Press. SHEPARD, R. N. (1984). Ecological constraints on internal representaton: Resonant kinematics of perceiving, imagining, thinking, and dreaming. Psychological Review, 91, 417-447.

SHEPARD, R. N., \& ZARE, S. L. (1983). Path-guided apparent motion. Science, 220, 632-634.

WATSON, A. B. (1986). Apparent motion occurs only between similar spatial frequencies. Vision Research, 26, 1727-1730.

WERTHEIMER, M. (1912). Experiemntalle Studien uber das Sehen von Bewegung [Experimental studies of motion perception]. Zeitschrift für Psychologie, 61, 161-265.

\section{NOTE}

1. In the treatment of mechanical systems, a distinction may be drawn between two different kinds of motion contexts. There are some contexts for which object motion is independent of all of the object's spatial attributes. In such contexts, objects move as if they were extensionless point particles located at their center of mass. A good example of this kind of context is gravitational freefall. In a vacuum, all bodies are equalized without regard to mass, shape, volume, or any other object attribute. All objects fall along parabolic trajectories at the same rate. $E x$ tended body motions are more general and occur in contexts in which some aspect of the object's extension in space determines the way in which it moves. Rolling wheels, spinning tops, and most realistic terrestrial motions occur as extended bodies. The motion of an extended body may be influenced by its shape, overall size, or orientation.

In apparent motion, there are no forces that determine how an object moves; the motion is purely kinematic, without a supporting dynamical structure. However, there is a natural division in apparent motion contexts in which a distinction between point particle and extended body arises. An apparent motion context is defined by the relative orientations in which stimuli are displayed. Thus, the point particle context is one in which nonorientable stimuli (circles or points of light) appear, or one in which an orientable stimulus is always presented in the same orientation. In this case, path perception will not interact with the spatial layout of the stimulus and perceived paths are straight lines. The extended body context is identified as one in which an orientable stimulus changes orientation in successive displays. This is not only the more general case, it is the case in which the shape of the stimulus introduces the possibility of curved paths into perception.

(Manuscript received July 27, 1987; revision accepted for publication November 17, 1987.) 